Special issue of the 3rd International Conference on Computational and Experimental Science and Engineering (ICCESEN 2016)

\title{
Designed Filter with CCII+ and Analysis of EEG for Epilepsy and Alzheimer
}

\author{
Ş. KITIŞ ${ }^{a, b *}, H$. APAYDIN ${ }^{b}$ AND R. GÜNTÜRKÜN ${ }^{c}$ \\ ${ }^{a}$ Sakarya University, Electric-Electronic Engineering Ph.D. student, Sakarya, Turkey \\ ${ }^{b}$ Dumlupınar University, Simav Vocational School, Kütahya, Turkey \\ ${ }^{c}$ Dumlupinar University, Technology Faculty, Kütahya, Turkey
}

\begin{abstract}
Biomedical signals are usually low amplitude and high bandwidth signals. EEG signals are very low amplitude (generally less than $300 \mu \mathrm{V}$ ) and very high bandwidth (range from 0.5 to $100 \mathrm{~Hz}$ ), too. EEG was used to assist in the diagnosis of brain damage, Alzheimer disease and Parkinson disease, and certain mental disorders. In this study amplifier and filters designed with CCII + and these simulations are performed with PSPICE program. Normal brain signal, the Alzheimer brain signal, epilepsy brain signal are compared, the shrinking hippocampus compared 25 years old normal brain, 75 years old normal brain and 75 years old with the Alzheimer brain.
\end{abstract}

DOI: 10.12693 /APhysPolA.132.423

PACS/topics: CCII+, Alzheimer, epilepsy, filter, amplifier

\section{Introduction}

The electroencephalogram (EEG) signals are small electrical potentials (generally less than $300 \mu \mathrm{V}$ ) produced by the brain [1-4]. The frequencies of these brain produced signals can range from 0.5 to $100 \mathrm{~Hz}$, and their characteristics are highly dependent on the degree of activity of the cerebral cortex $[4,5]$.

EEG is used in the evaluation of brain disorders. Most commonly it is used to show the type and location of the activity in the brain during a seizure. It also is used to evaluate people who are having problems associated with brain function. These problems might include confusion, tumors, long-term difficulties with thinking or memory, or weakening of specific parts of the body [6].

EEG is a key tool in the diagnosis and management of epilepsy and other seizure disorders. It is also used to assist in the diagnosis of brain damage and disease, mental retardation, sleep disorders, degenerative diseases such as Alzheimer disease and Parkinson disease, and certain mental disorders [7].

Electrocorticography (ECoG) is a technique that uses subdural electrodes to record neural activity directly from the cortical surface and has been widely used in patients with epilepsy to localize the origin of epileptic seizures [8-11].

\section{Materials and methods}

\subsection{EEG measurements and designed filters, amplifiers} with CCII for EEG, ECoG

EEG measurements generally are made with electrodes placed over the skull (Fig. 1).

\footnotetext{
*corresponding author; e-mail: sukru.kitis@dpu.edu.tr
}

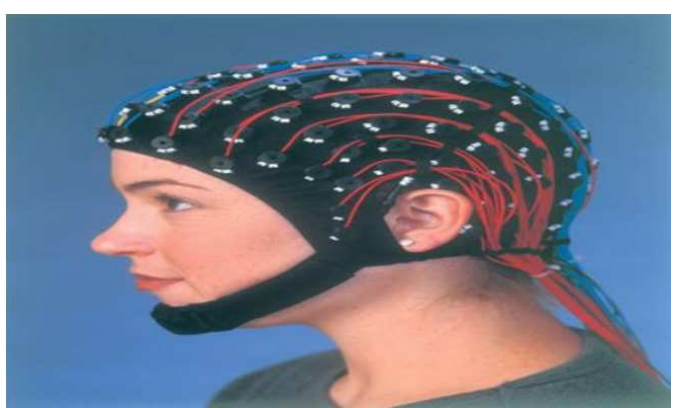

Fig. 1. EEG hood [29].

EEG measurements rarely are made with electrodes placed in the skull for disease progressed people (Fig. 2). These measurements are called epidural ECoG and subdural ECoG.
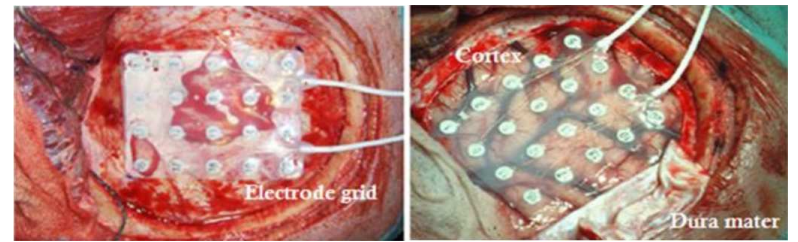

Fig. 2. Epidural ECoG and subdural ECoG [11].

Filters and amplifier devices are very important for EEG and the others biomedical measurements. Biomedical signals are very low amplitude. EEG signals have low amplitude and high bandwith. Therefore filters are important for EEG signals, too. Signals are received from the skull and treated preamplifier, low pass filter, high pass filter, etc. (Fig. 3).

Current conveyors (CCII + ) have a greater linearity, input voltage interval and frequency performance than the circuits with operational amplifiers (OPAMP). Therefore, they have recently begun to be quite often used in the electronic circuits. Designs with CCII + structure 


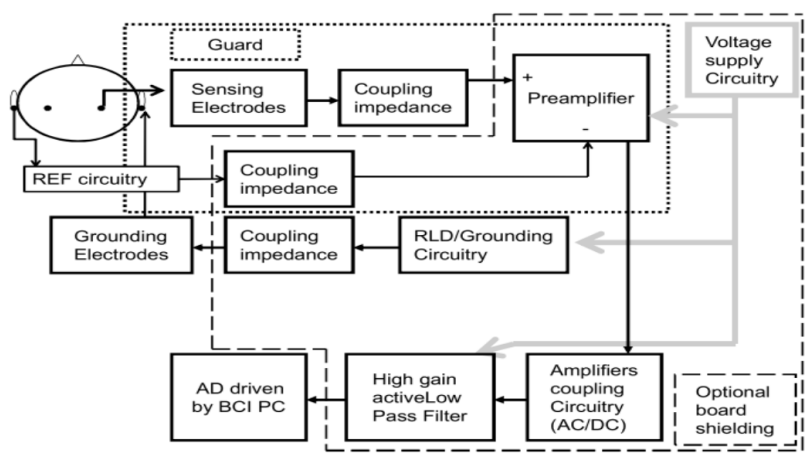

Fig. 3. Block diagram of EEG [12].

have become widespread in amplifier circuits, integrator circuits, filter circuits, and differentiator circuits [13, 2124].

In this study designed filters and amplifiers with $\mathrm{CCII}+$ for biomedical devices and these simulations are performed with PSPICE programs (Figs. 4-7).

\subsection{PSPICE results}

Instrumentation amplifier, high pass filter, low pass filter and inverting amplifier devices with $\mathrm{CCII}+$ designed and performed at PSPICE programs (Figs. 4-7).

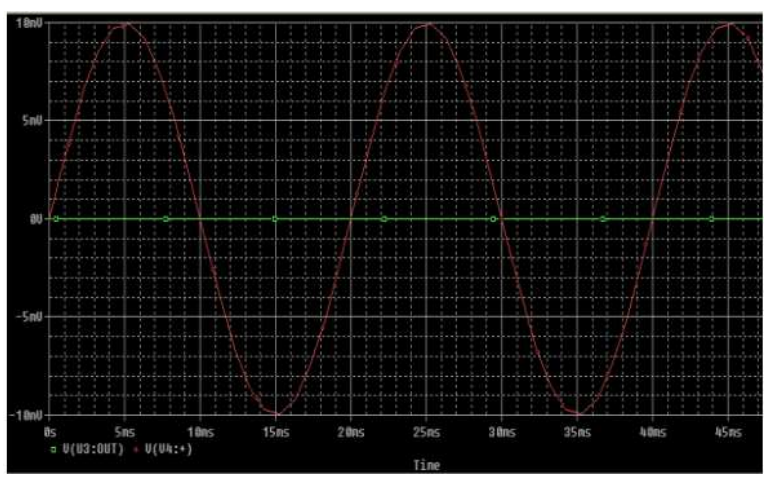

Fig. 4. Instrumentation amplifier with $\mathrm{CCII}+$ at PSPICE simulation.

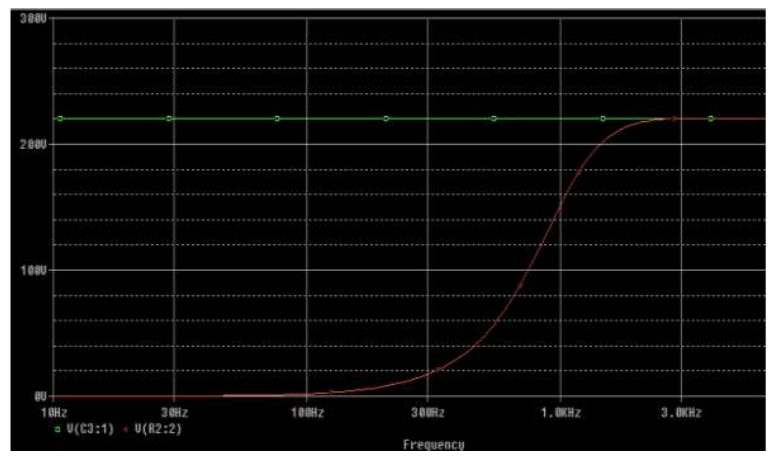

Fig. 5. High-pass filter with CCII+ at PSPICE simulation.

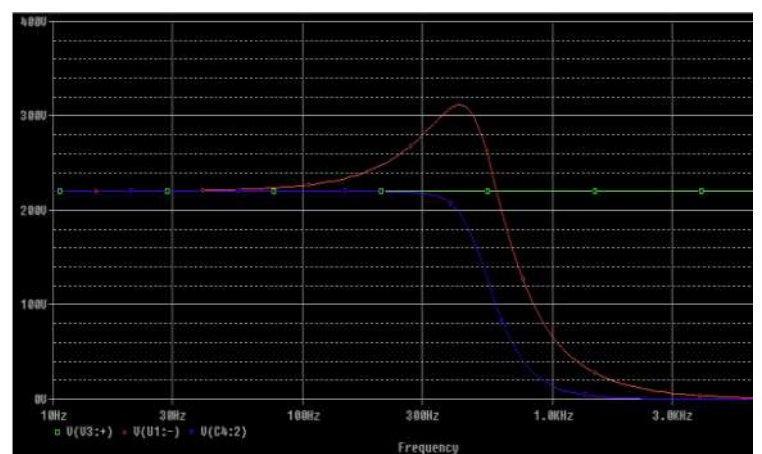

Fig. 6. Low-pass filter with CCII + at PSPICE simulation.

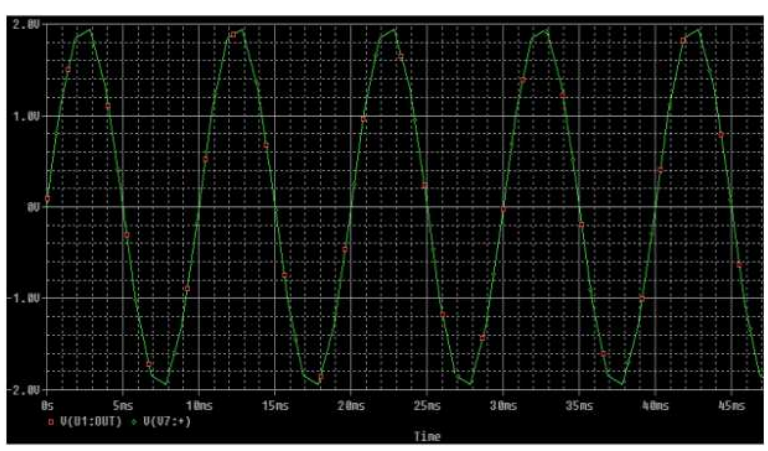

Fig. 7. Inverting amplifier with CCII + at PSPICE simulation.

\subsection{A model designed and practised for $E E G$ measurements with CCII}

In this study a model was designed (Fig. 8) and practised for EEG measurements with CCII+.

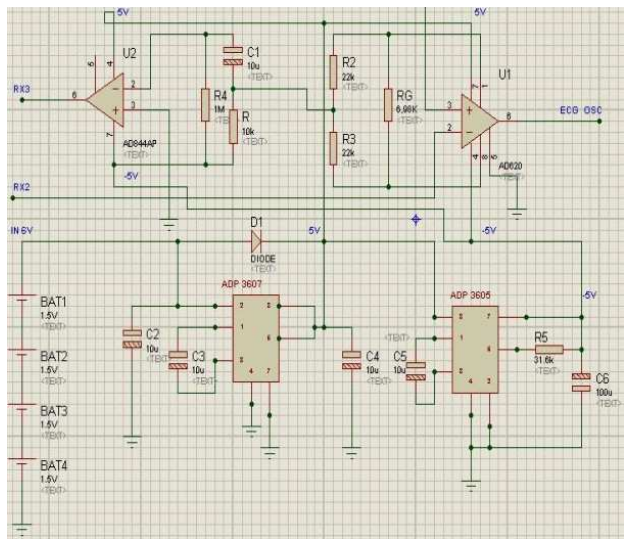

Fig. 8. A model designed for EEG measurements.

\subsection{Causes of epilepsy and Alzheimer disease, EEG signals and treatment of Alzheimer disease}

\subsubsection{Hippocampus of epileptic and Alzheimer patients}

A curved structure nestled deep within the brain, the hippocampus plays a major role in forming, storing, and 
processing memories. The hippocampus becomes somewhat smaller as a part of normal ageing, as shown by the comparison between the hippocampus in Figs. 9, 10.
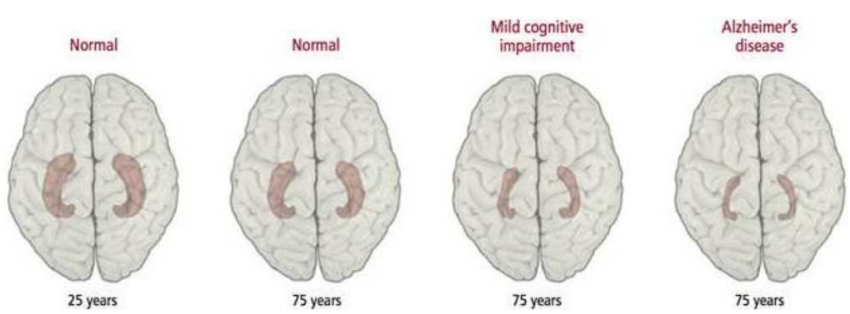

Fig. 9. The shrinking of hippocampus [14].
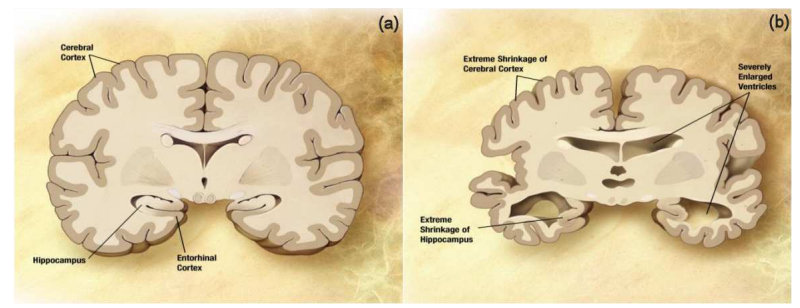

Fig. 10. Diagrams of a brain before (a) and after (b) the development of Alzheimer disease [15].

\subsubsection{EEG signals}

Normal brain signal, Alzheimer brain signal, and epilepsy brain signal are showed in Figs. 11-13.

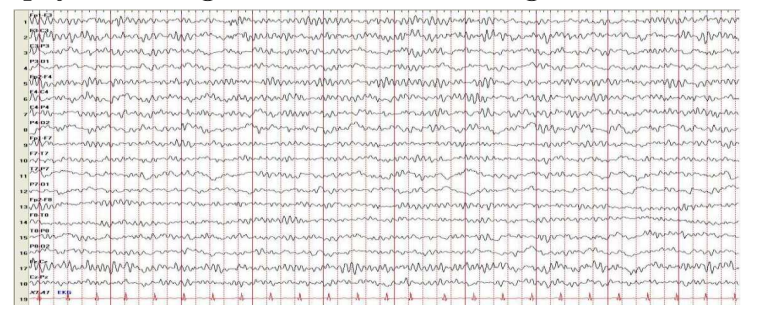

Fig. 11. Normal brain signal [16].

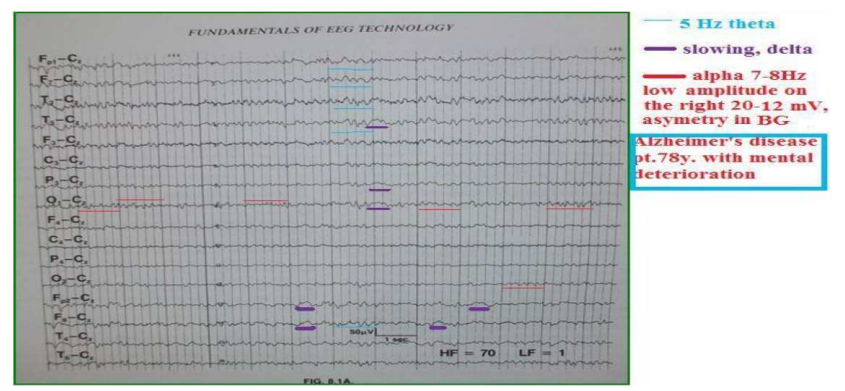

Fig. 12. Alzheimer brain signal [17].

\subsubsection{Preventing Alzheimer disease}

The course of Alzheimer disease is not the same in every person, but symptoms seem to develop over the same general stages. In most people with Alzheimer, symptoms first appear in their mid-60s. Scientists know that Alzheimer progresses on a spectrum with three stages an early, preclinical stage with no symptoms; a middle stage of mild cognitive impairment (MCI); and a final stage of Alzheimer dementia. At this time one cannot

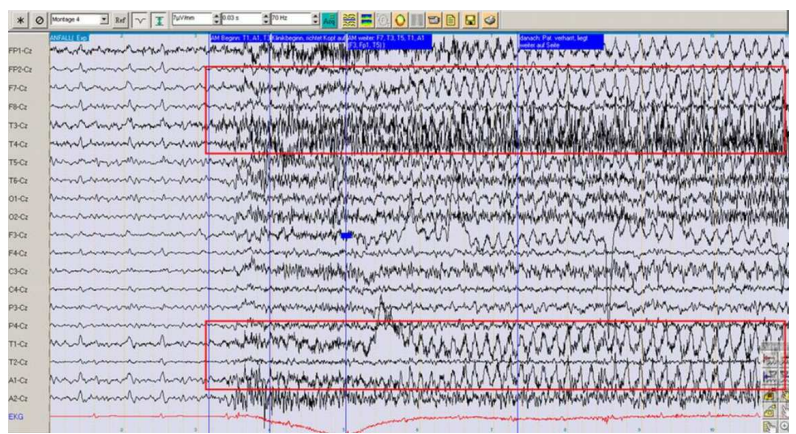

Fig. 13. Epilepsy brain signal [18].

predict with significant level of certainty if MCI will develop Alzheimer disease [19].

Promising research shows that you can reduce your risk of Alzheimer and other dementias through a combination of healthy habits, including right nutrition, exercising, staying mentally and socially active, and keeping stress in check (Fig. 14). By leading a brain-healthy lifestyle, you may be able to prevent the symptoms of Alzheimer disease and slow down, or even reverse, the process of deterioration [20].

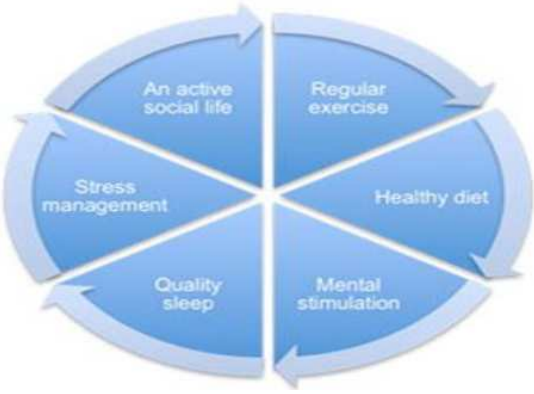

Fig. 14. Preventing Alzheimer disease.

\section{Results and discussion}

Current conveyors $(\mathrm{CCII}+)$ have a greater linearity, input voltage interval and frequency performance than the circuits with OPAMP. Instrumentation amplifier, high pass filter, low pass filter and inverting amplifier devices with CCII + are designed and performed at PSPICE programs. These devices will be used at EEG measurements and diagnosis of epilepsy and Alzheimer disease, so that fighting with these brain disorders. Abnormal changes in frequency and voltage for epilepsy will be determined with high pass filter at this model and alpha waves' changes in frequency for Alzheimer disease will be determined with low pass filter at this model, too.

\section{References}

[1] J.D. Bronzino, in: Biomedical Engineering Handbook, Ed. J.D. Bronzino, 2nd ed., CRC Press, 2000, p. 1.

[2] J.G. Webster, Medical Instrumentation Application and Design, III ed., Wiley, 1998. 
[3] P. Sovilj, M. Milovanović, D. Pejić, M. Urekar, Z. Mitrović, Meas. Sci. Rev. 14, 270 (2014).

[4] Brain In A Vat, a neuroscience research digest, Method of the Month: EEG, September 2007.

[5] M. Abeles, M. Goldstein, Proc. IEEE 65, 762 (1977).

[6] Emedicinehealth, Electroencephalography (EEG) 2016.

[7] The Encyclopedia of Surgery Ce-Fi, Electroencephalography.

[8] A. Palmini, A. Gambardella, F. Andermann, F. Dubeau, J.C. da Costa, A. Olivier, D. Tampieri, P. Gloor, F. Quesney, E. Andermann, E. Paglioli, E. Paglioli-Neto, L. Coutinho, R. Leblanc, H.I. Lim, Ann. Neurol. 37, 476 (1995).

[9] D. Zumsteg, H.G. Wieser, Epilepsia 41, S55 (2000).

[10] K. Takaura, N. Tsuchiya, N. Fujii, NeuroImage $\mathbf{1 2 4}$ 557 (2016).

[11] Jang's Lab, Minimal Invasive Environment-Brain Interface.

[12] G. Gargiulo, P. Bifulco, R.A. Calvo, M. Cesarelli, C. Jin, A. van Schalk, in: Biomedical Circuits Systems Conf., BioCAS 2008, Baltimore (USA), IEEE.

[13] A. Sedra, K.C. Smith, IEEE Trans. Circuit Theory 17, 132 (1970)

[14] Helpguide, Recognizing Alzheimer's Disease.
[15] UCSF Memory and Aging Center, Alzheimer's Disease.

[16] Somatosphere, EEG, January 2014.

[17] Studyblue, Degenerative Diseases.

[18] Otto-von-Guericke-Universität, Universitätsklinik für Neurologie, Epilepsieambulanz.

[19] National Institute on Aging, What Happens to the Brain in Alzheimer's Disease?, August 2017.

[20] Helpguide, Preventing Alzheimer's Disease.

[21] M. Challal, M. Boulakroune, Acta Phys. Pol. A 130, 9 (2016).

[22] M.E. Başak, F. Kaçar, Acta Phys. Pol. A 130, 223 (2016).

[23] M. Challal, M. Dehmas, A. Azrar, R. Aksas, M. Trabelsi, Acta Phys. Pol. A 128, B-71 (2015).

[24] X. Jiang, P. Ren, C. Luo, Cognit. Comput. 8, 828 (2016)

[25] X. Liu, Y. Zeng, T. Zhang, B. Xu, Cognit. Comput.8, 967 (2016).

[26] Y. Yang, I. Bloch, S. Chevallier, J. Wiart, Cognit. Comput. 8, 505 (2016).

[27] L. Cao, J. Li, Y. Xu, H. Zhu, C. Jiang, Cognit. Comput. 8, 228 (2016).

[28] H. Zhao, J. Ren, Cognit. Comput. 8, 246 (2016). 